\title{
Aspectos Clínicos e Terapêuticos da Osteomielite Vertebral por Fungos - Análise Secundária de Dados
}

\section{Clinical and Therapeutic Features of Fungal Vertebral Osteomyelitis. A Secondary Data Analysis}

\author{
Giovannini Cesar Figueiredo ${ }^{(1)}$, Evânia Claudino Queiroga de Figueiredo ${ }^{(2)}$, José Tavares-Neto ${ }^{(3)}$
}

\section{RESUMO}

Objetivo: avaliar os dados bibliométricos e as manifestações clínicoterapêuticas da osteomielite vertebral por fungos, retirados dos relatos de casos publicados e disponíveis nos bancos de dados eletrônicos. Método: revisão sistemática, com análise secundária de dados, considerando os casos comprovados, do banco de dados Medline, Embase e Lilacs, e da busca ativa das referências, entre 1966 e 2004. Resultados: houve a tendência de crescimento de publicações de casos de osteomielite vertebral por fungos no período $\left(\mathrm{R}^{2}=0,5518\right)$, com a maioria ocorrendo após 1990 (218/318 [68,5\%]). Os relatos provenientes da América do Norte predominaram (148/318 [46,5\%]). O agente etiológico mais comum foi Candida sp (131/318 [41,2\%]), seguido de Aspergillus sp (102/318 [32,1\%]). O tempo compreendido entre o aparecimento dos sintomas e o diagnóstico variou de uma semana a 9,6 anos, com média de 24,4 $\pm 41,6$ semanas. A coluna lombo-sacra foi o segmento mais afetado (188/318 [59,1\%]), sendo a coluna cervical acometida em apenas $24 / 318(7,5 \%)$ descrições. O evento infeccioso ocorreu com mais freqüência na combinação L2 + L3 (35/318 [11\%]), sendo L2 e L3 as vértebras mais lesionadas (84/318 [26,4\%], cada uma). O tratamento cirúrgico foi realizado em $194 / 307(63,2 \%)$ casos com clara notificação, dos quais a artrodese foi necessária em 91/307 (29,6\%). Foi observada uma tendência da utilização de compostos azólicos, em substituição à anfotericina $\mathrm{B}$, no tratamento da infecção, entre os períodos de 1966-1989 (10/37) e 1990-2004 (40/63). Conclusão: a osteomielite vertebral por fungos é um evento mais freqüentemente publicado após 1990, sendo necessária a máxima atenção para que o seu diagnóstico não passe despercebido tanto tempo, por falta de uma solicitação de pesquisa micológica rotineira ao serviço de microbiologia geral.

\begin{abstract}
Objectives: to assess the bibliometric, clinical and therapeutic features of fungal vertebral osteomyelitis case reports, retrieved from electronic databases. Methods: systematic review with secondary data analysis done on proven cases from Medline, Embase and Lilacs databases and active search of referenced published reports, since 1966 until 2004. Results: there was a growing trend from 1966 to $2004\left(R^{2}=0.5518\right)$ in the 318 retrieved cases of fungal vertebral osteomyelitis: $218 / 318$ (68.5\%) of them from 1990 on. Reports with diagnosis from North America prevailed (148/318 [46.5\%]). The most common causative agent was Candida spp (131/318 [41.2\%]), followed by Aspergillus spp (102/318 [32.1\%]). Time elapsed between symptom appearance and diagnosis ranged from one week to 9.6 years, mean $24.4 \pm 41.6$ weeks. The lumbar-sacral spine was the most affected segment (188/318 [59.1\%]), and the cervical spine was involved in only $24 / 318$ descriptions (7.5\%). The combination L2+L3 was the most frequent infectious event (35/318 [11\%]); L2 and L3 were the most affected vertebrae $(84 / 318[26.4 \%])$. Surgical treatment was carried out in $194 / 307(63.2 \%)$ cases. Arthrodesis was the surgical procedure performed in $91 / 307$ (29.6\%) with clear notification. There was a growing trend of azole compound utilization, in substitution to amphotericin B, between 1966-1989 (10/37) and 1990-2004 (40/63). Conclusion: fungal vertebral osteomyelitis has been more frequently reported since the 1990s. Great attention to the possibility of this diagnosis is necessary so that the fungal vertebral infection is not missed due to the lack of a routine mycological search in a general microbiology laboratory.
\end{abstract}

Keywords: osteomyelitis, fungi, spine.

Palavras-chave: osteomielite, fungo, coluna vertebral.

Parte integrante de Tese de Doutorado em Medicina e Saúde, do Programa de Pós-Graduação em Medicina e Saúde da Universidade Federal da Bahia (UFBA), Universidade Estadual da Paraíba (UEPB) e Universidade Federal de Campina Grande (UFCG). Recebido em 13/06/06. Aprovado, após revisão, em 22/1 1/06. 1. Mestre e doutor em Medicina e Saúde pela Universidade Federal da Bahia (UFBA). Professor Titular de Clínica Traumatortopédica da Universidade Estadual da Paraíba (UEPB).

2. Mestre em Medicina e Saúde pela UFBA. Professora assistente de Reumatologia da Universidade Federal de Campina Grande (UFCG).

3. Professor livre-docente, diretor da Faculdade de Medicina da Bahia (Fameb) da Universidade Federal da Bahia (UFBA)

Endereço para correspondência: Giovannini Cesar Figueiredo, Rua José Bonifácio, 67/501, CEP 58100-640, Campina Grande, PB, Brasil, telefone (83) 3321-2142, fax (83) 3341-4666, e-mail: giocesar@uol.com.br. 


\section{INTRODUÇÃO}

A capacidade dos fungos de causar doenças invasivas é muito bem documentada. As espécies Candida constituem, hoje, o quarto microrganismo mais isolado nas hemoculturas de pacientes internados nos Estados Unidos, atrás apenas do Staphylococcus aureus, estafilococos coagulase-negativa e enterococos ${ }^{(1)}$. De acordo com Cone et al ${ }^{(2)}$, citando vários estudos epidemiológicos do Estado de Virgínia ${ }^{(3)}$, os índices de candidemia nosocomial aumentaram em dez vezes entre 1978 e 1984, duplicaram entre 1980 e 1990, com a tendência contínua de expansão ${ }^{(4)}$. Os avanços da medicina relacionados ao transplante de órgãos, a utilização de implementos cirúrgicos artificiais e as imunodeficiências são os motivos da instalação da fungemia. Nesse grupo, dos indivíduos que desenvolvem a fungemia (20\%), em $15 \%$ o órgão a distância-alvo da infecção é o osso ou a articulação. Nos adultos com osteomielite por disseminação hematogênica, seja por bactéria ou fungo, os corpos e espaços vertebrais são os sítios mais comuns.

O diagnóstico da infecção osteoarticular causada por fungo é difícil, devido aos indicadores clínicos inespecíficos e pouco sensíveis. A ausência de estudos mais sistematizados e com maior casuística justifica essa revisão sistemática, que conclui com um alerta para a necessidade de se considerar o fungo como agente etiológico no diagnóstico diferencial da osteomielite vertebral.

\section{PACIENTES E MÉTODO}

Realizaram-se uma revisão sistemática, com análise secundária de dados, de casos comprovados de osteomielite vertebral por fungos, levantados dos bancos de dados eletrônicos Medline, Embase e Lilacs, e busca ativa das referências destes artigos, entre 1966 e 2004. Todos os casos descritos nos idiomas português, espanhol, italiano, francês e inglês foram incluídos. Foram excluídos os casos cujos agentes, antes considerados fungos, foram reclassificados como bactérias fungiformes. Para aumentar a sensibilidade da revisão, utilizamos níveis de estratégia de pesquisa para identificação de relatos de casos no Medline sugerido por Dickersin et al $(1994)^{(5)}$. O primeiro passo preconizou a inclusão de termos relacionados à pesquisa da condição principal (e.g.: fungi and osteomyelitis; fungi and spondylodiscitis or spondylitis), o segundo passo preconizou a inclusão de termos relacionados à intervenção (e.g.: spondylodiscitis and fluconazole; vertebra or vertebrae and amphotericin $B$ ) e o terceiro passo preconizou a inclusão de termos relacionados com a metodologia dos ensaios clínicos (e.g.: fungi and case report). Os gêneros de fungos utilizados como termos de busca foram retirados de tratado de micologia médica (Kern \& Blevins, 1999)(6).

Os relatos foram examinados, com os dados bibliométricos, clínicos e terapêuticos, sendo extraídos e registrados em um formulário de sistematização criado previamente para esse fim. Antes da análise dos dados, cada descrição foi sistematizada por dois grupos de observadores (um ortopedista e um reumatologista), com a supervisão do professor-orientador (infectologista), e os pontos discordantes foram analisados em conjunto por ambos os observadores para inclusão, se existisse consenso.

Os dados clínico-terapêuticos foram avaliados de forma descritiva e comparativa. Para as variáveis nominais foram utilizados o $\chi^{2}$ de Pearson, corrigidos por Yates ou pelo teste exato de Fisher. O teste não-paramétrico de Mann-Whitney foi utilizado para as variáveis contínuas sem distribuição normal. Para os dados bibliométricos, também foi utilizada a linha de tendência com grau de correlação.

\section{RESULTADOS}

\section{BIBLIOMETRIA}

Todos os 318 relatos de casos de osteomielite vertebral por fungos foram sistematizados em um formulário com elementos ou critérios clínico-terapêuticos (Figueiredo \& Tavares-Neto $)^{(7)}$. A maioria dos casos (148/318 [46,5\%]) pertencia à classe $\mathrm{D}$, isto é, eles apresentaram entre $51 \%$ e $70 \%$ das informações requeridas pelo formulário de sistematização. Os relatos restantes pertenciam à classe $\mathrm{C}$ $(128 / 318[40,2 \%])$ e E $(41 / 318$ [12,9\%]), com $71 \%$ a $90 \%$ e menos do que $50 \%$ da informação, respectivamente. Apenas $1 / 318(0,3 \%)$ tinham $91 \%$ a $99 \%$ (classe B) e nenhum tinha $100 \%$ (classe $A$ ) das informações solicitadas. A média de casos por artigo variou de um a 16, com média de 1,5 .

Houve a linha de tendência ascendente, de 1966 a 2004 $\left(\mathrm{R}^{2}=0,5518\right)$, no número de casos relatados de osteomielite vertebral por fungos; $218 / 318(68,5 \%)$ de $1990 \mathrm{em}$ diante (Figura 1). Os relatos provenientes da América do Norte predominaram (148/318 [46,5\%]); dos quais, 145/148 (98\%) foram dos Estados Unidos. A seguir vieram os países europeus $(106 / 318[33,3 \%])$, a Ásia $(29 / 318[9,1 \%])$, a África $(22 / 318[6,9 \%])$, a Oceania $(9 / 318[2,8 \%])$ e a América do Sul $(4 / 318$ [1,2\%]), dos quais $2 / 4$ (50\%) do Brasil $^{(8,9)}$. A maior freqüência de publicações advindas dos Estados Unidos em relação aos outros países não se alterou, 


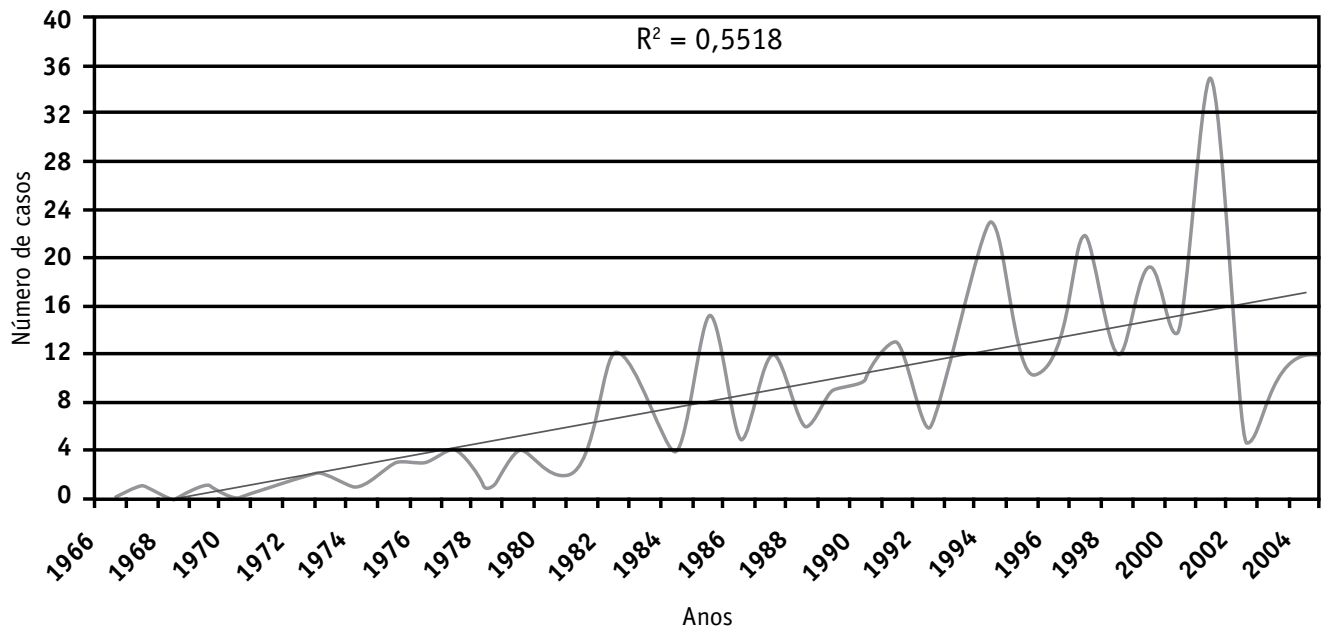

\footnotetext{
Osteomielite vertebral por fungos

Linear (osteomielite verbal por fungos)
}

Figura 1. Distribuição dos casos de osteomielite por fungos, segundo o período de tempo.

quando comparadas as publicações em períodos separados ([EUA/outros países] vs [1966-1989/1990-2004] $=[53 / 47] /[92 / 126] ;$ Pearson $\left.\chi^{2}=3,22[p>0,07]\right)$. O periódico que mais publicou casos de osteomielite vertebral por fungos foi o Clinical Infectious Diseases com 27/318 casos $(8,5 \%)$, seguido pelo Journal of Spinal Disorders com 20/318 (6,3\%); Spine com 18/318 (5,7\%); The Journal of Bone and Joint Surgery Am com 15/318 (4,7\%); Journal of Infection com 11/318 (3,5\%); The American Journal of Medicine e The Journal of Rheumatology com 10/318 $(3,1 \%)$ relatos cada.

A respeito dos relatos que informaram a especialidade ou o serviço dos autores, $40 / 285$ (14\%) tinham a participação de pelo menos um reumatologista (Tabela 1); somente $23 / 318$ (8\%) tinham exclusivamente reumatologistas como autores.

\section{DADOS CLÍNICOS}

O sexo masculino superou o feminino $(215 / 67,6 \%$ vs $103 / 32,4 \%)$. A idade do paciente variou de 1,5 mês a 88 anos, com média de 43,8 $\pm 20,2$ anos. O fator de associação mais freqüente (Tabela 2), segundo os relatos que continham a informação, foi a neoplasia $(48 / 268$ $[17,9 \%])$, seguido pelo uso de drogas injetáveis $(35 / 268$ $[13,1 \%])$, corticoterapia $(31 / 268[11,6 \%])$, diabetes $(26 / 268[9,7 \%])$, laparotomia $(25 / 268[9,3 \%])$, cateterização prolongada $(20 / 268[7,5 \%])$, transplante de órgãos $(18 / 268[7,5, \%])$ e quimioterapia $(17 / 268[6,3 \%])$. Trinta
TABELA 1

FREQÜÊNCIA DE PARTICIPAÇÃO DAS ESPECIALIDADES NOS RELATOS DE CASOS

\begin{tabular}{|c|c|c|c|}
\hline Especialidade & $\mathbf{n}$ & $\%$ & \% válida \\
\hline Ortopedia & 85 & 26,7 & 29,8 \\
\hline Infectologia & 83 & 26,1 & 29,1 \\
\hline Reumatologia & 40 & 12,6 & 14,0 \\
\hline Clínica médica & 36 & 11,3 & 12,6 \\
\hline Radiologia & 34 & 10,7 & 11,9 \\
\hline Neurocirurgia & 33 & 10,4 & 11,6 \\
\hline Microbiologia & 31 & 9,7 & 10,9 \\
\hline Patologia & 31 & 9,7 & 10,9 \\
\hline Pediatria & 18 & 5,7 & 6,3 \\
\hline Hematologia & 17 & 5,3 & 6,0 \\
\hline Cirurgia geral & 11 & 3,5 & 3,9 \\
\hline Micologia & 9 & 2,8 & 3,2 \\
\hline Neurologia & 7 & 2,2 & 2,5 \\
\hline Bacteriologia & 4 & 1,3 & 1,4 \\
\hline Medicina tropical & 4 & 1,3 & 1,4 \\
\hline Nefrologia & 4 & 1,3 & 1,4 \\
\hline Psiquiatria & 4 & 1,3 & 1,4 \\
\hline Gastroenterologia & 3 & 0,9 & 1,0 \\
\hline Laboratório & 3 & 0,9 & 1,0 \\
\hline Pneumologia & 3 & 0,9 & 1,0 \\
\hline Cirurgia cardiovascular & 2 & 0,6 & 0,7 \\
\hline Cirurgia torácica & 2 & 0,6 & 0,7 \\
\hline Cirurgia vascular & 2 & 0,6 & 0,7 \\
\hline Dermatologia & 2 & 0,6 & 0,7 \\
\hline Farmacologia & 2 & 0,6 & 0,7 \\
\hline Imunologia & 2 & 0,6 & 0,7 \\
\hline Oftalmologia & 2 & 0,6 & 0,7 \\
\hline Parasitologia & 2 & 0,6 & 0,7 \\
\hline Anestesiologia & 1 & 0,3 & 0,3 \\
\hline Cardiologia & 1 & 0,3 & 0,3 \\
\hline Cirurgia bucomaxilofacial & 1 & 0,3 & 0,3 \\
\hline Cirurgia cabeça e pescoço & 1 & 0,3 & 0,3 \\
\hline Endocrinologia & 1 & 0,3 & 0,3 \\
\hline Oncologia & 1 & 0,3 & 0,3 \\
\hline Otorrino & 1 & 0,3 & 0,3 \\
\hline Transplante & 1 & 0,3 & 0,3 \\
\hline UTI & 1 & 0,3 & 0,3 \\
\hline Sem informação & 33 & 10,4 & - \\
\hline
\end{tabular}


TABELA 2

FATORES DE ASSOCIAÇÃO DESCRITOS NOS RELATOS DE CASOS DE OSTEOMIELITE FÚNGICA

\begin{tabular}{|c|c|c|c|}
\hline Fator de associação & $\mathbf{n}$ & $\%$ & \% válida \\
\hline Neoplasia & 48 & 15,1 & 17,9 \\
\hline Usuário de drogas & 35 & 11,0 & 13,1 \\
\hline Corticoterapia & 31 & 9,7 & 11,6 \\
\hline Diabetes & 26 & 8,2 & 9,7 \\
\hline Laparotomia & 25 & 7,9 & 9,3 \\
\hline Cateterização & 20 & 6,3 & 7,5 \\
\hline Transplante & 18 & 5,7 & 6,7 \\
\hline Quimioterapia & 17 & 5,3 & 6,3 \\
\hline Antibioticoterapia & 15 & 4,7 & 5,6 \\
\hline Laminectomia & 13 & 4,1 & 4,8 \\
\hline Tuberculose & 12 & 3,8 & 4,5 \\
\hline Alcoolismo & 11 & 3,5 & 4,1 \\
\hline DPOC & 9 & 2,8 & 3,4 \\
\hline Insuficiência renal & 8 & 2,5 & 3,0 \\
\hline Aids & 7 & 2,2 & 2,6 \\
\hline Doença granulomatosa crônica & 6 & 1,9 & 2,2 \\
\hline Prótese cirúrgica & 6 & 1,9 & 2,2 \\
\hline Hepatite & 5 & 1,6 & 1,9 \\
\hline Cirurgia vascular & 3 & 0,9 & 1,1 \\
\hline Desnutrição & 3 & 0,9 & 1,1 \\
\hline Lúpus & 3 & 0,9 & 1,1 \\
\hline Prematuridade & 3 & 0,9 & 1,1 \\
\hline Septicemia & 3 & 0,9 & 1,1 \\
\hline Trauma & 3 & 0,9 & 1,1 \\
\hline Gota & 2 & 0,6 & 0,7 \\
\hline Sarcoidose & 2 & 0,6 & 0,7 \\
\hline Toracotomia & 2 & 0,6 & 0,7 \\
\hline Abortamento & 1 & 0,3 & 0,4 \\
\hline Anemia & 1 & 0,3 & 0,4 \\
\hline Aplasia medular & 1 & 0,3 & 0,4 \\
\hline Asma & 1 & 0,3 & 0,4 \\
\hline Aspergilose pulmonar & 1 & 0,3 & 0,4 \\
\hline Cardiopatia reumática & 1 & 0,3 & 0,4 \\
\hline Cirurgia ortopédica & 1 & 0,3 & 0,4 \\
\hline Cirurgia urológica & 1 & 0,3 & 0,4 \\
\hline Endocardite & 1 & 0,3 & 0,4 \\
\hline Esplenectomia & 1 & 0,3 & 0,4 \\
\hline Glomerulonefrite & 1 & 0,3 & 0,4 \\
\hline Obesidade mórbida & 1 & 0,3 & 0,4 \\
\hline Pneumonia & 1 & 0,3 & 0,4 \\
\hline Queimadura & 1 & 0,3 & 0,4 \\
\hline
\end{tabular}

e seis pacientes eram imunologicamente competentes.

A via hematogênica foi considerada a provável forma de contaminação em 287/307 (93,5\%) dos casos com a notificação. A osteomielite vertebral por Aspergillus esteve mais associada a fator torácico (Pearson $\chi^{2}=11,4$ $[\mathrm{p}<0,001])$ e a transplante de órgão (Pearson $\chi^{2}=6,6$ $[p<0,02])$ do que àquela infectada por Candida. Por outro lado, o acometimento por Candida esteve mais associado ao consumo de drogas injetáveis do que o acometimento por Aspergillus (Pearson $\chi^{2}=13,7[p<0,0003]$ ). A dor foi relatada em $100 \%$ dos casos e havia comprometimento neurológico quando do diagnóstico em 90/210 (42,9\%) dos casos válidos. A síndrome febril ocorreu em 106/198 $(53,5 \%)$ dos casos com clara notificação. A contagem leucocitária do sangue, em 103 descrições, variou de $800 \mathrm{~mm}^{3}$ a $30.300 / \mathrm{mm}^{3}$, com média de $9.834 \mathrm{~mm}^{3} \pm$ $4.384 \mathrm{~mm}^{3}$, mediana de $9.100 \mathrm{~mm}^{3}$ e moda de $8.700 \mathrm{~mm}^{3}$.
Em 142 registros disponíveis, a velocidade de sedimentação de hemácias (VSH) variou de $2 \mathrm{~mm}$ a $190 \mathrm{~mm}$ na primeira hora, média de $76,1 \mathrm{~mm} \pm 34,8 \mathrm{~mm}$, mediana de $72 \mathrm{~mm}$ e moda de $50 \mathrm{~mm}$.

O tempo compreendido entre o aparecimento dos sintomas e o diagnóstico variou de uma semana a 9,6 anos, com média de 24,4 $\pm 41,6$ semanas, mediana de 13 semanas e moda de 9 semanas. $\mathrm{O}$ atraso foi $<2$ semanas em $8 / 191$ pacientes (4,2\%), 2-6 semanas em 42/191 (22\%), $>6$ semanas- 6 meses em 96/19l (50,3\%), > 6-12 meses em 30/19l (15,7\%) e > l ano em 15/191 (7,8\%).

A ressonância nuclear magnética (RNM) e a cintilografia identificaram a lesão lítica vertebral em $100 \%$ dos casos e o RX foi insensível em 16/192 (8,3\%) dos casos válidos.

$\mathrm{Na}$ maioria dos relatos, a infecção ocorreu na coluna lombo-sacra $(188 / 318[59,1 \%])$ e torácica $(148 / 318$ $[46,5 \%])$. A coluna cervical foi acometida em apenas 24/318 (7,5\%) descrições. A combinação L2 + L3 foi o evento mais comum $(35 / 318$ [11\%]), seguida por L3 + L4 (23/318 [7,2\%]), L4 + L5 (22/318 [6,9\%]), L1 + L2 $(21 / 318[6,6 \%])$ e Tl1 + Tl2 (18/318 [5,7\%]). A vértebra mais atingida (Figura 2 ) foi L2 e L3 $(84 / 318$ [26,4\%], cada uma), L4 (70/318 [22\%]) e Ll (56/318 [17,6\%]).

\section{MICROBIOLOGIA}

A cultura para fungos foi positiva em 272/278 (97,8\%) casos com informação disponível. A positivação da cultura para fungo ocorreu durante a primeira pesquisa micológica em 166/193 (86\%) casos; em 26/193 (13,5\%) ocorreu durante a segunda pesquisa, e $1 / 193(0,5 \%)$ ocorreu somente na terceira pesquisa micológica. Após a confirmação da presença do fungo, este foi considerado como agente etiológico verdadeiro já na primeira positivação em $188 / 193$ casos $(97,4 \%)$. O exame histopatológico resultou negativo em 29/138 (21\%) relatos. Nos 115 casos que foram realizados, tanto a cultura como o histopatológico, $80(69,5 \%)$ positivaram ambos, enquanto o diagnóstico alcançado pela cultura, em vez do histopatológico, totalizou 29/35 (82,9\%) [Fisher's exact test; $p>0,05$ ].

$\mathrm{O}$ agente causal mais comum foi Candida $s p(131 / 318$ [41,2\%]), dos quais $C$. albicans foi a espécie predominante $(82 / 131[62,6 \%])$, seguida por $C$. tropicalis $(24 / 131$ $[18,3 \%])$, C. glabrata $(12 / 131[9,2 \%])$, C. parapsilosis (8/131 [6,1\%]), C. species (3/131 [2,3\%]), C. guilliermondi e C. stellatoidea (1/131 [0,8\%], cada uma). O segundo gênero mais freqüente foi Aspergillus sp (102/318 $[32,1 \%])$. A. fumigatus foi a espécie predominante $(71 / 102$ 


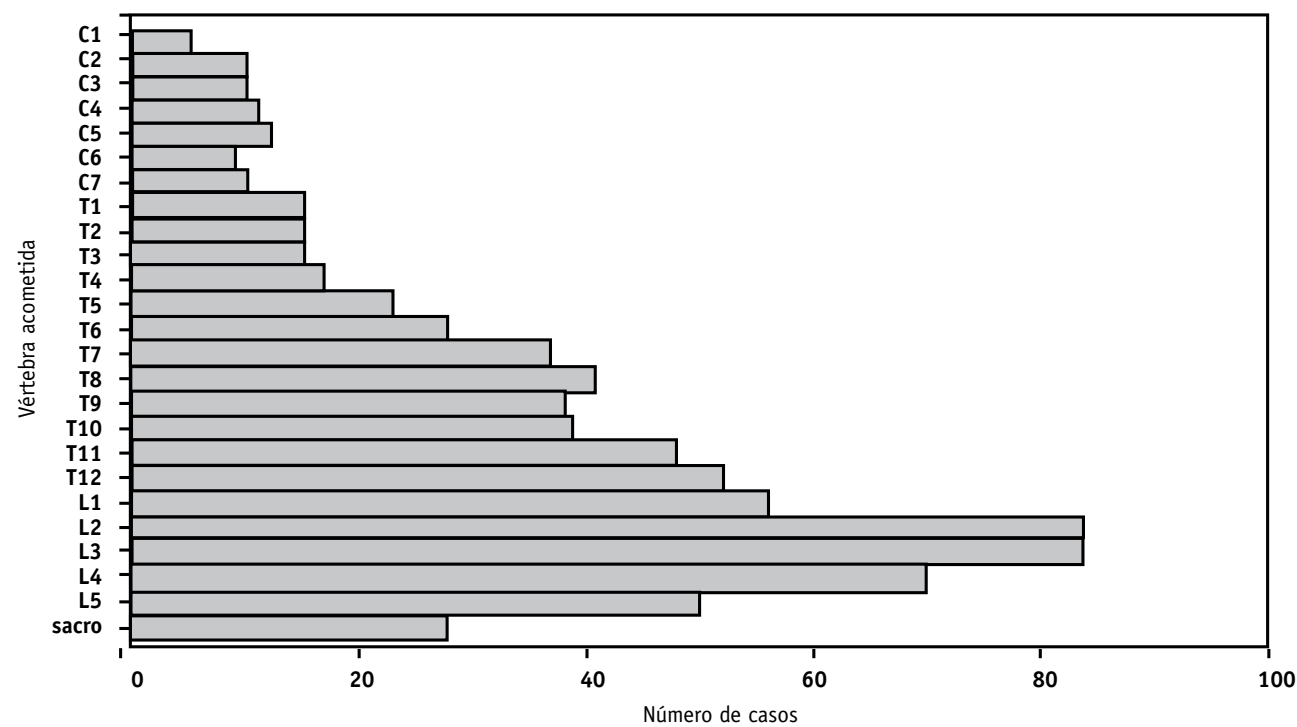

Figura 2. Distribuição do acometimento vertebral.

$[69,6 \%])$, seguida por A. flavus (13/102 [12,7\%]), A. species (11/102 [10,8\%]), A. terreus (4/102 [3,9\%]), A. niger (3/102 [2,9\%]), A. nidulans e $A$. versicolor $(1 / 102$ [1\%], cada uma). Outros agentes relatados foram Coccidioides (23/318 [7,3\%]), Cryptococcus (22/318 [7\%]), Blastomyces (15/318 [4,7\%]), Pseudallescheria (8/318 [2,5\%]), Histoplasma $(5 / 318[1,6 \%])$, Blastochyzomyces $(3 / 318[0,9 \%])$, Paracoccidioides e Penicillium (2/318 [0,6], cada uma). Acremonium, Drechslera, Fusarium, Phialemoniume Nattrassia foram diagnosticados somente uma única vez $(0,3 \%)$.

\section{PROCEDIMENTOS TERAPÊUTICOS}

O tratamento cirúrgico foi indicado em 194/307 $(63,2 \%)$ dos casos válidos, com a artrodese sendo necessária em 91/307 (29,6\%) casos com clara notificação (Tabela 3). A osteomielite vertebral por Aspergillus exigiu mais a indicação cirúrgica do que aquela infectada por Candida (Pearson $\left.\chi^{2}=18,9[p<0,00002]\right)$.

Os dados sobre o tempo de manutenção da terapia antimicótica estavam disponíveis em 153 relatos, variando de uma semana a três anos, com média de $21,3 \pm 22,4$ semanas, mediana de 13 semanas e moda de seis semanas. A anfotericina B foi utilizada em 238/297 (80,1\%) descrições; sendo em 138/238 (58\%) associada a outro antimicótico. A dose total de anfotericina B, em 126/238 casos, variou de $27 \mathrm{mg}$ a $6.000 \mathrm{mg}$, média de $1.700 \mathrm{mg} \pm$ $1.100 \mathrm{mg}$, mediana de $1.500 \mathrm{mg}$, e moda de $2.000 \mathrm{mg}$.
TABELA 3

FREQÜÊNCIA DO PROCEDIMENTO TERAPÊUTICO

\begin{tabular}{lccc}
\hline Procedimento & $n$ & $\%$ & \% válida \\
\hline Aspiração percutânea & 14 & 4,4 & 4,6 \\
\hline Excisão e drenagem & 61 & 19,2 & 19,9 \\
Discectomia & 14 & 4,4 & 4,6 \\
\hline Discectomia + corporectomia & 11 & 3,5 & 3,6 \\
Costotransversectomia & 3 & 0,9 & 1,0 \\
\hline Artrodese & 91 & 28,6 & 29,5 \\
a. Discectomia & 71 & 22,3 & 23,1 \\
b. Corporectomia & 18 & 5,7 & 5,8 \\
c. Costotransversectomia & 2 & 0,6 & 0,6 \\
Somente medicamentoso & 113 & 35,5 & 36,8 \\
\hline Sem dados & 11 & 3,5 & - \\
Total & 318 & 100,0 & 100,0 \\
\hline
\end{tabular}

Anfotericina $B$ foi administrada em um período que variou de duas a 13 semanas, média de 7,6 $\pm 3,2$ semanas, mediana de oito semanas. Os compostos azólicos foram empregados em 145/297 (48,8\%) descrições, sendo em 56/145 $(38,6 \%)$ o itraconazol. A dosagem diária do itraconazol, em $39 / 56(69,6 \%)$ relatos, variou de $100 \mathrm{mg}$ a $900 \mathrm{mg}$, média de $333,3 \mathrm{mg} \pm 206,9 \mathrm{mg}$, mediana $300 \mathrm{mg}$, e moda $200 \mathrm{mg}$. A dosagem diária de fluconazol, em 45/54 (83,3\%) relatos, variou de $100 \mathrm{mg}$ a $900 \mathrm{mg}$, média de $336,7 \mathrm{mg} \pm 183,2 \mathrm{mg}$, mediana e moda de $400 \mathrm{mg}$. O itraconazol foi usado em um período que variou de uma a 91 semanas, média de 
$51,6 \pm 26,8$ semanas, mediana de 52 semanas. O fluconazol foi administrado em um período que variou de quatro a 87 semanas, média de 25,3 $\pm 22,6$ semanas, mediana de 19,5 semanas. Houve uma linha ascendente da utilização dos compostos azólicos, em substituição à anfotericina B (Figura 3), entre os períodos 1966-1989 (10/37) e 1990-2004 (40/63) [Pearson $\left.\chi^{2}=4,5 ; p<0,04\right)$.

$\mathrm{O}$ desfecho foi considerado como cura sem recorrência em 178/268 (66,4\%) relatos; melhora e/ou recorrência em $52 / 268$ (19,4\%); e ocorreram 38/268 (14,2\%) óbitos durante o tratamento devido a complicações sistêmicas. A cura não esteve associada ao tempo decorrido até o diagnóstico (Mann-Whitney $\mathrm{U}=1480[p>0,2])$. Não houve seqüela em 173/192 (90,1\%) casos que continham a informação. O tempo de seguimento após a cura considerada variou de dois meses a 3,8 anos, média de 25,5 $\pm 29,2$ meses, mediana de 16,5 meses e moda de 12 meses.

\section{DISCUSSÃO}

A osteomielite vertebral por fungos é um evento mais freqüentemente publicado a partir de 1990. Certamente, a prevalência de casos provenientes dos Estados Unidos constitui um viés de diagnóstico ou publicação. $\mathrm{O}$ fungo é um patógeno de distribuição mundial.

A maior freqüência de Candida $s p$ e Aspergillus $s p$ como agentes etiológicos de osteomielite vertebral parece estar relacionada ao fato de Candida ser um colonizador comum da pele e Aspergillus, do trato respiratório. Os relatos dispersos de agentes como Acremonium ${ }^{(10)}$, Drechslera $^{(11)}$, Fusarium ${ }^{(12)}$, Phialemonium ${ }^{(13)}$ e Nattrassia ${ }^{(14)}$, comprovam, de fato, que qualquer fungo poderia causar espondilodiscite infecciosa.

Atenções especiais devem ser dadas aos pacientes submetidos a procedimentos invasivos no hospital e, ocasionalmente, uma dorsolombalgia sem resolução torna-se um confuso problema. Na maioria dos casos, a espondilodiscite fúngica acontece como complicação de uma fungemia. $\mathrm{O}$ atraso do diagnóstico da osteomielite vertebral por fungos, apontado por esse estudo, foi de vários meses, variando de poucos dias até muitos anos, um fato também relatado por Hendrickx et $a l^{(15)}$. O curso clínico da infecção foi indolente. Metade dos casos não apresentou mudanças na temperatura corporal e a contagem leucocitária (média, mediana e moda) esteve abaixo do limite considerado como positivo para a presença de infecção. $\mathrm{O}$ exame físico e os recursos de imagem, como radiografias convencionais da coluna, cintilografia óssea, tomografia computadorizada (TC) e ressonância nuclear magnética (RNM) deram suporte ao argumento inespecífico para o diagnóstico da osteomielite vertebral por fungos. As planigrafias foram ineficientes para o diagnóstico precoce, com a RNM constituindo o teste de escolha, acrescentando ao diagnóstico a avaliação para a possível necessidade do

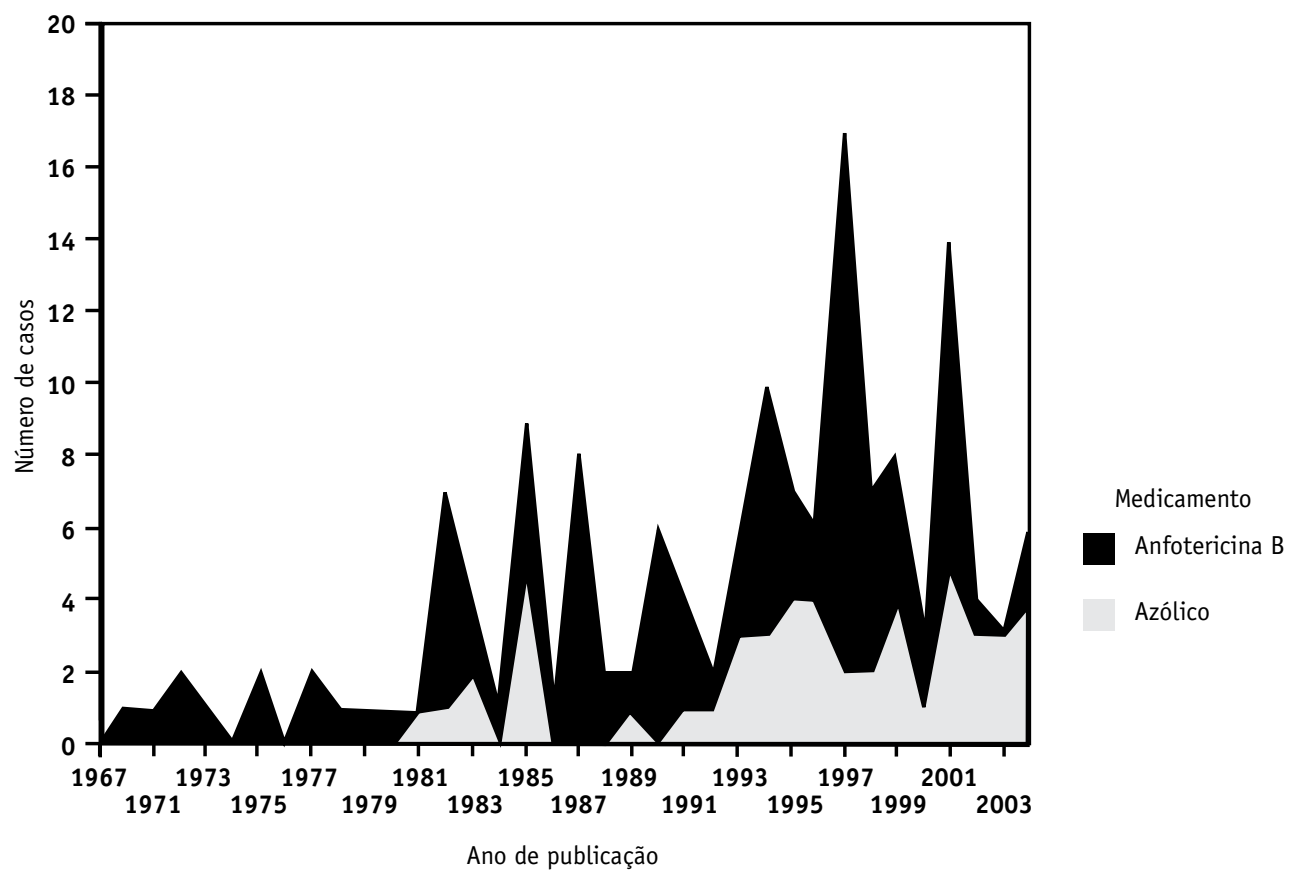

Figura 3 - Distribuição do uso de anfotericina B e compostos azólicos, de acordo com o tempo. 
manejo cirúrgico, consideração já apontada por McHenry

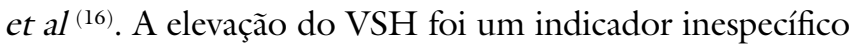
que pode ser considerado para monitorar a resposta clínica ao tratamento da osteomielite fúngica vertebral. Ensaios antigênicos para o rápido diagnóstico de infecções fúngicas invasivas podem ser utilizados, como Polymerase Chain Reaction (PCR), Galactomannan (GM) e Western-blot (WB) para a detecção de anticorpos e dos metabólitos fúngicos D-arabinitol e $(1,3)$ - $\beta$-D-glucan ${ }^{(17,18)}$.

Vários protocolos de tratamento foram indicados para a osteomielite vertebral por fungos. Alguns autores prescreveram somente drogas antimicóticas ${ }^{(19)}$. Outros realizaram artrodese após corporectomia radical ${ }^{(20)}$. Entretanto, o procedimento seriado mais freqüentemente observado foi a composição de drenagem do abscesso, discectomia e debridamento de todo tecido infectado, artrodese com um sistema de estabilização e auto-enxertia óssea, e administração intravenosa de anfotericina B em curto período, seguida por administração oral de composto azólico por longo período. Os limites extremos da dose total de anfotericina $\mathrm{B}$ foram conseqüência de intolerância à droga e à recorrência da infecção. Por muitos anos, a anfotericina B tem sido a droga de eleição para o tratamento das infecções fúngicas invasivas. Apesar da prática de se manter a terapia até a resolução dos sinais e sintomas clínicos da infecção, pareceu evidente que a anfotericina $\mathrm{B}$ deveria ser infundida, consensualmente, dentro de um limite de $2.000 \mathrm{mg}$ durante oito semanas, enquanto o fluconazol ou itraconazol deveria ser empregado em uma dose diária de $400 \mathrm{mg}$ por, pelo menos, seis meses. Por causa das desvantagens da nefrotoxicidade e efeitos adversos à infusão, vários complexos lipídicos de anfotericina B têm sido desenvolvidos ${ }^{(21)}$. Mesmo assim, sua prescrição tem sido reservada para pacientes que não podem tolerar a formulação convencional, devido ao custo dessa medicação; se bem que a anfotericina B lipossomal também é relatada com insucesso terapêutico ${ }^{(22)}$. Novas drogas com promessa de eficiência têm sido relatadas em

\section{REFERÊNCIAS}

1. Kao AS, Brandt MS, Pruitt WR, et al: The epidemiology of candidemia in two United States cities: results of populationbased active surveillance. Clin Infect Dis 29: 1164-70, 1999.

2. Cone LA, Byrd RG, Potts BE, Wuesthoff M: Diagnosis and treatment of Candida vertebral osteomyelitis: clinical experience with a short course therapy of amphotericin B lipid complex. Surg Neurol 62: 234-7, 2004.

3. Morrison AJ, Freer CV, Searcy MA, Landry SM, Wenzel RP: Nosocomial blood stream infections: secular trends in statewide surveillance program in Virginia. Infect Control 7: 550-3, 1976. estudos comparativos com seguimento ${ }^{(23)}$, e outros para melhorar a resistência do hospedeiro aos fungos ${ }^{(24)}$.

A pesquisa por fungos, apesar de não ser atribuição especial do reumatologista, deve ser solicitada e cobrada por este. Os resultados da análise bibliométrica mostraram que, nos relatos e casos de osteomielite vertebral por fungos, a autoria exclusiva e absoluta de cada especialidade envolvida pode ser considerada pequena, já que sua participação é compartilhada com outros especialistas, principalmente o ortopedista, o infectologista e o reumatologista. Se por um lado isso demonstra que essa patologia tem enfoque multidisciplinar, por outro revela a necessidade de maior envolvimento de todas as especialidades relacionadas ao aparelho locomotor para estudar o problema. Tudo isso pode levar à informação e ao conhecimento setorizados insuficientes, necessários à estruturação de critério investigativo, provocando viés no diagnóstico e publicação de casos de osteomielite vertebral por fungos.

Portanto, é atribuição especial do especialista investigador compartilhar a melhor atenção com o microbiologista, para que o diagnóstico da espondilodiscite fúngica não seja protelado por ausência da pesquisa micológica rotineira (como é feita com a bacteriana), em um serviço geral de microbiologia.

\section{CONCLUSÕES}

A osteomielite vertebral por fungos é um evento mais freqüentemente publicado a partir de 1990, e os agentes etiológicos mais relatados foram o gênero Candida e Aspergillus. O tempo compreendido entre o aparecimento e os sintomas foi longo (média de 24 semanas). A infecção por Candida esteve relacionada ao consumo de drogas e por Aspergillus, à transplante de órgãos e a fator torácico prévio. Os antimicóticos mais utilizados foram a anfotericina B e os azólicos itraconazol e fluconazol, porém há uma tendência de substituir a anfotericina B pelos azólicos no tratamento medicamentoso da osteomielite vertebral por fungos.

Declaramos a inexistência de conflitos de interesse.

4. Beck-Sague CM, Jarvis WR and the National Nosocomial Infections Surveillance System: Secular trends in the epidemiology of nosocomial fungal infections in the United States, 1980-1990. J Infect Dis 167: 1247-51, 1993.

5. Dickersin K, Scherer R, Lefebvre C: Identifying relevant studies for systematic reviews. Br Med J 309: 1286-91, 1994.

6. Kern MA, Blevins KS: Micologia Médica. Texto \& Atlas. 2. ed. São Paulo: Editorial Premier, 1999.

7. Figueiredo GC, Tavares-Neto J: Estruturação de um banco de dados para análise secundária de informações em relatos ou série de casos. Rev Bras Ortop 36: 407-11, 2001. 
8. Marchiori E, Pereira AA, Aymoré IL: Blastomicose vertebral - evolução de 13 anos. Radiol Bras 15: 117-9, 1982.

9. Milazzo LC, Veloso GA: Forma localizada de paracoccidioidomicose na coluna vertebral. Rev Bras Ortop 27: 150-2, 1992.

10. Noble RC, Salgado J, Newell SW, Goodman NL: Endophtalmitis and lumbar diskitis due to Acremonium falciforme in a splenectomized patient. Clin Infect Dis 24: 277-8, 1997.

11. Drouhet E, Guilmet D, Kouvalchouk JF, et al: First human case of Drechslera longirostrata mycosis. Spondylodiscitis complicating prosthesis endocarditis. Treatment with combined ketoconazole and amphotericin B. Nouv Press Med 11: 3631-5, 1982.

12. Moschovi M, Trimis G, Anastasopoulos J, Kanariou M, Raftopoulou A, Tzortzatou-Stathopoulou F: Subacute vertebral osteomyelitis in a child with diabetes mellitus associated with Fusarium. Pediatr Int 46: 740-2, 2004.

13. Magnon KC, Jalbert M, Padhye AA: Osteolytic phaeohyphomycosis caused by Phialemonium obovatum. Arch Pathol Lab Med 117: 841-3, 1993.

14. Willinger B, Kopetzky G, Harm F, et al: Disseminated infection with Nattrassia mangiferae in an immunosuppressed patient. J Clin Microbiol 42: 478-80, 2004.

15. Hendrickx L, Van Wijngaerden E, Samson I, Paetermans WE: Candidal vertebral osteomyelitis: Report of six patients, and a review. Clin Infect Dis 32: 527-33, 2001.

16. McHenry MC, Easley KA, Locker GA: Vertebral osteomyelitis: long-term outcome for 253 patients from 7 Cleveland-area hospitals. Clin Infect Dis 34: 1342-50, 2004.
17. Alexander BD: Diagnosis of fungal infection: new technologies for the mycology laboratory. Transpl Infect Dis 4 Suppl 3: 32-7, 2002.

18. Reiss E, Obayashi T, Orle K, Yoshida M, Zancope-Oliveira RM: Non-culture based diagnostic tests for mycotic infections. Med Mycol 38: 147-59, 2000.

19. Derkinderen P, Bruneel F, Bouchaud O, Regnier B: Spondylodiscitis and epidural abscess due to Candida albicans. Eur Spine J 9: 72-4, 2000

20. Vaishya S, Sharma MS: Spinal Aspergillus vertebral osteomyelitis with extradural abscess: Case report and review of literature. Surg Neurol 61: 551-5, 2004.

21. Linden PK: Amphotericin B lipid complex for the treatment of invasive fungal infections. Expert Opin Pharmacother 4: 2099110,2003

22. Stratov I, Korman TM, Johnson PD: Management of Aspergillus osteomyelitis: report of failure of liposomal amphotericin $B$ and response to voriconazole in an immunocompetent host and literature review. Eur J Clin Microbiol Infect Dis 22: 277-83, 2003.

23. Boucher HW, Groll AH, Chiou CC, Walsh TJ: Newer systemic antifungal agents: pharmacokinetics, safety and efficacy. Drugs 64: 1997-2020, 2004.

24. Ivanovska N, Hristova A: Treatment with oxoglaucine can enhance host resistance to Candida albicans infection of mice with adjuvant arthritis. Diagn Microbiol Infect Dis 38: 17-20, 2000. 